\title{
ROCSAT-1 Satellite Observations of Magnetic Anomaly Density Structures During the Great Magnetic Storm of July 15-16, 2000
}

\author{
Chin S. Lin ${ }^{1, *}$, Huey-Ching Yeh ${ }^{2}$, and Shin-Yi Su ${ }^{2}$ \\ (Manuscript received 18 July 2001, in final form 15 August 2001)
}

\begin{abstract}
Large scale ion density structures were observed by ROCSAT-1 at 630 $\mathrm{km}$ altitude over the South Atlantic anomaly (SAA) region during the great magnetic storm of July 15-16, 2000. The observed density structures were confined within $30^{\circ}$ in longitude east of the SAA, and co-rotated with the SAA for over 8 hours. The magnetic anomaly density structures (MADS) observed during several successive orbits in the nighttime sector, contained density holes and density pile-ups adjacent to each other. The density holes had a density drop of one to two orders of magnitude below the ambient level and a spatial size of $800-1800 \mathrm{~km}$ along the satellite path. The density pile-ups typically spanned a spatial size of $2000 \sim 3000 \mathrm{~km}$ were characterized with a density enhancement, large density fluctuations and upward drift velocity. In contrast to density pile-ups, density holes did not exhibit noticeable density fluctuations nor upward drift velocity. A common feature of large poleward drifts was noticeable inside both density hole and pile-up structures. The ion drift measurements thus indicate that ions inside the density holes drifted away in the poleward direction rather than the upward direction. The successive observations of density holes on six orbits suggest that the density hole might be initially created near the center of SAA region and then expanded equatorward. We interpret the magnetic anomaly density pile-up to be the ionospheric F2 peak layer raised to the satellite altitude by storm enhanced eastward electric fields. It is speculated that energetic particle precipitation might have occurred in the region of magnetic anomaly density structures, increasing locally ionospheric conductivity in the $\mathbf{E}$ region and producing eastward polarization electric fields in the precipitation region. The combination of localized polarization electric fields and storm time electric fields at low latitudes in the nightside might be responsible for the ion drifts and the associated anomalous density structures observed by ROCSAT-1 in the F region.
\end{abstract}

\footnotetext{
${ }^{1}$ National Space Program Office, Hsin-Chu, Taiwan

2 Institute of Space Science, National Central University, Chung-Li, Taiwan

${ }^{*}$ Corresponding author address: Dr. Chin S. Lin, 8F, 9 Prosperity 1st Road, Science-based Industrial Park, Hsin-Chu, Taiwan; E-mail: cslin@nspo.gov.tw
} 


\section{(Key words: Magnetic anomaly, South Atlantic anomaly, Density hole, Density pile-up, Magnetic storm)}

\section{INTRODUCTION}

During the great magnetic storm of July 15-16, 2000, the ROCSAT-1 satellite measured unusual large scale ion density structures at low-latitudes $\left(<35^{\circ}\right)$ in the southern hemisphere. The ion density structures observed by ROCSAT- 1 contained regions of large scale ion density depletion and the adjacent density enhancement. Ion density holes characterized by a density decrease of more than an order of magnitude from the ambient were found to span over a distance from several hundreds to $2000 \mathrm{~km}$ along the satellite track. Such large size density holes are similar to equatorial ion density depletions first reported by Greenspan et al. (1991) using the DMSP satellite thermal plasma measurements during the great magnetic storm of March 1989. DMSP detected equatorial density holes after the ring current reached its maximum intensity. In addition DMSP measured a sharp density enhancement poleward of equatorial density depletions (Greenspan et al. 1991). Foster and Rich (1998) also used DMSP data to study equatorial ion depletions during another severe magnetic storm. It appears that largescale density depletions are rare and might occur only during great storms when the disturbance dynamo effects reach the low-latitude ionosphere.

Because DMSP detected equatorial density holes near the South Atlantic anomaly (SAA), it has been speculated that the development of equatorial density holes might be related to the SAA (Greenspan et al. 1991; Foster and Rich, 1998). In this paper we present ROCSAT-1 observational evidence relating the occurrence of anomalous density structures with the SAA. Specifically the satellite data have been used to show that large scale density holes and density pile-ups were confined near the SAA and co-rotated with the SAA. Importantly, unlike the polar orbiting DMSP satellite observing the latitudinal structures, the ROCSAT-1 satellite with a low inclination orbit provided the longitudinal profile of the SAA anomalous density structures never obtained before.

The magnetic storm of July 15-16, 2000 has been considered to be one of the most intense storms in the last century. Figure 1, a time history plot of the Kp and Dst indices, shows the storm evolution. The time intervals of the seven orbits studied in this paper are marked by dashed lines with letters from $a$ to $g$ in the top panel. The Kp index started to increase dramatically from 1200 UT July 15, 2000 and remained high until 0600 UT July 16, 2000. As indicated by Dst index, the storm main phase started in 2040 UT on July 15, 2000 when the magnetic activity was already very intense $(\mathrm{Kp}=9)$. The storm activity reached its peak intensity in $2200 \mathrm{UT}$ (Dst $\approx-300 \mathrm{nT}$ ). While Dst remained near its lowest value, ROCSAT-1 detected the first anomalous density structure (orbit $b$ ). The ROCSAT-1 satellite observed additional anomalous density structures until 0700 UT on the following five orbits, including the last three orbits (orbit $e, f$ and $g$ ) during the storm recovery phase (Dst $=-200$ ). We present the satellite observations during these orbits in Section 2, discuss and summarize the results in Section 3. 


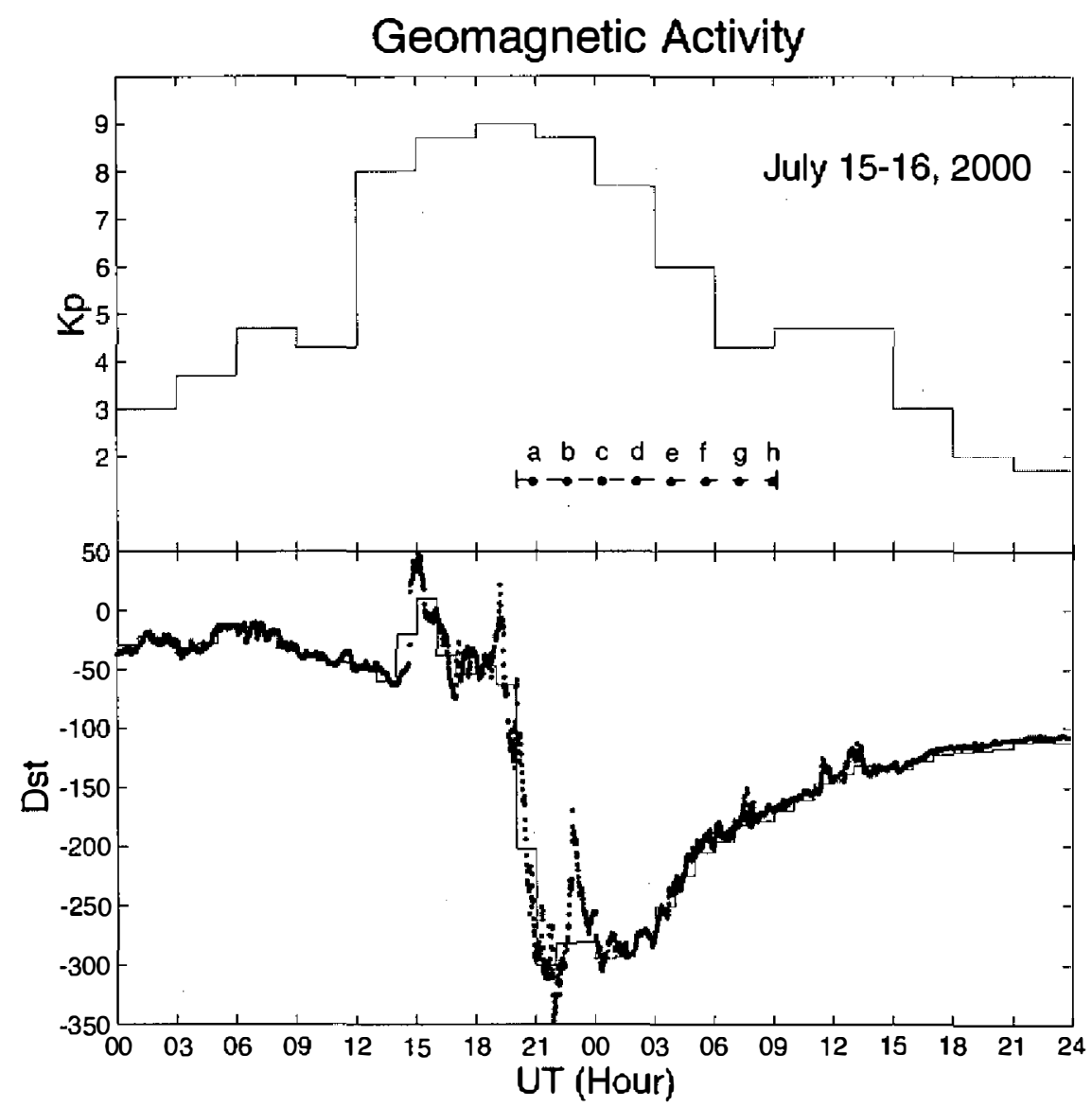

Fig. 1. UT variations of Kp and Dst indices for July $15-16,2000$. The intervals of ROCSAT-1 orbits assigned with letters from $a$ to $h$ are shown in the top panel.

\section{SATELLITE OBSERVATIONS}

The ROCSAT-1 satellite launched in 1999 had a circular orbit with an altitude near 630 $\mathrm{km}$ and an orbital inclination angle of $35^{\circ}$. Onboard the ROCSAT-1 satellite, the Ionospheric Plasma and Electrodynamics Instrument (IPEI) payload contained an ion trap to measure ion density, two drift meters to measure ion drift velocities in the cross-track directions, and a retarding potential analyzer to infer the plasma parameters of major ion constituents including temperature and ram velocity (Chang et al.1999; Yeh et al. 1999). In this paper we concentrated on ion density data from the ion trap and drift velocity data from the two ion drift meters.

Figure 2 shows the measured profile of ion density and velocities when ROCSAT- 1 de- 
tected the first anomalous density structure. The ion density measured by the ion trap sensor decreased gradually from $10^{6} \mathrm{~cm}^{-3}$ (around 2200 UT) to about $3 \times 10^{5} \mathrm{~cm}^{-3}$ (around 2219 UT) as ROCSAT-1 moved from the afternoon sector to the dusk sector (top panel of Fig. 2). Around 2219 UT ROCSAT-1 began to detect a sharp density decrease which reached as low as $4 \times 10^{4}$ $\mathrm{cm}^{-3}$ at $2222 \mathrm{UT}$. After the density drop, the total ion density recovered abruptly at 2222:15 UT to $10^{6} \mathrm{~cm}^{-3}$, slightly higher than the density level before the density drop was detected. Noticeably the measured ion density showed large fluctuations and a narrow density bubble with a low density of $2 \times 10^{4} \mathrm{~cm}^{-3}$ at $2223 \mathrm{UT}$. The density bubble was observed with a duration of $26 \mathrm{~s}$ or equivalently a spatial width of about $200 \mathrm{~km}$. Figure 2 displays another fine bubble around 2226 UT with a spatial width estimated to be around $15 \mathrm{~km}$. After the end of

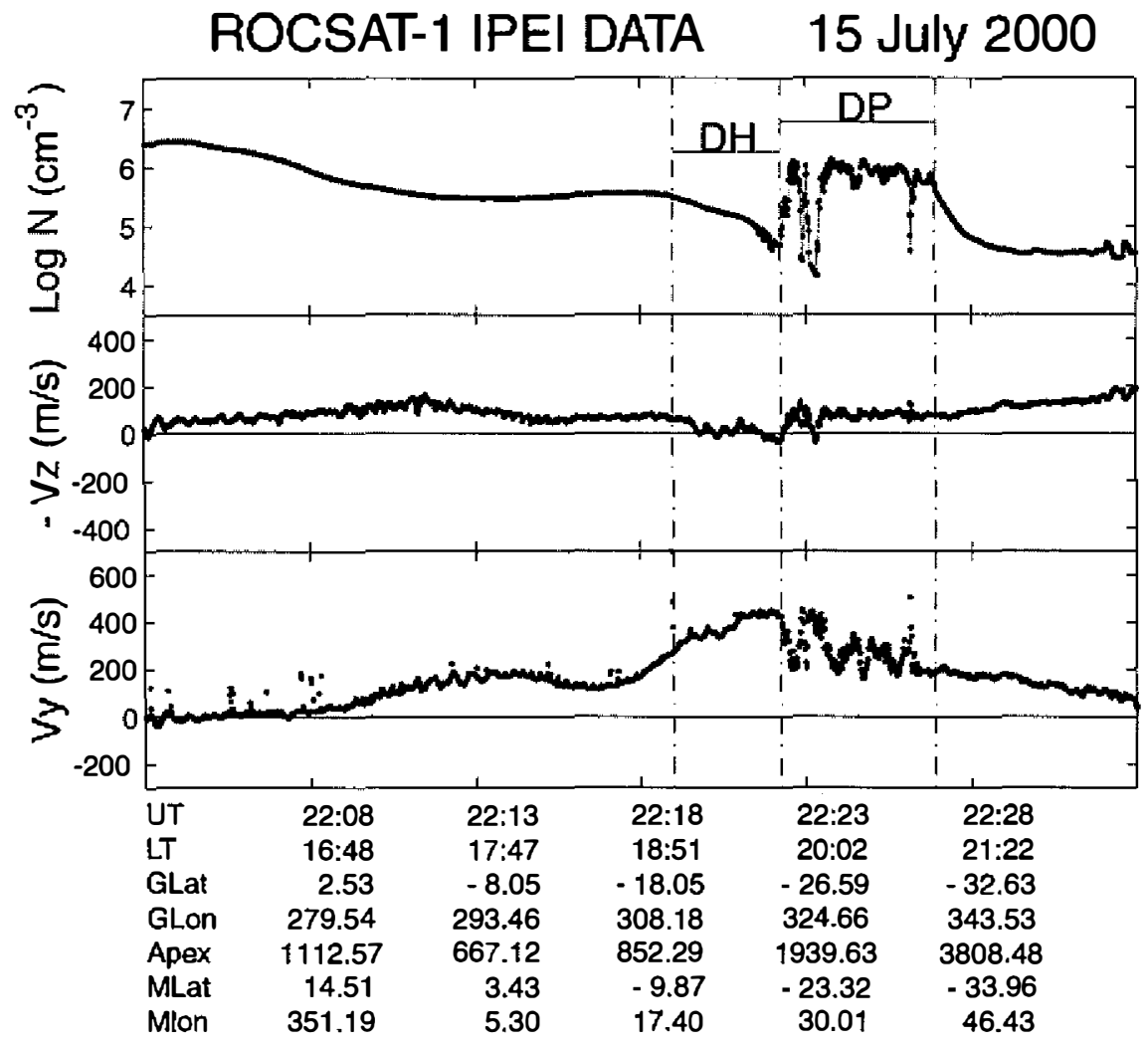

Fig. 2. ROCSAT-1 IPEI observations of total ion density (top), vertical velocity $-\mathrm{V}_{z}$ (middle), and horizontal $\mathrm{V}_{\mathrm{y}}$ velocity (bottom). In the spacecraft coordinate positive $\mathrm{V}_{z}$ points toward Earth (downward) and positive $\mathrm{V}_{\mathrm{y}}$ points anti-parallel to the orbital normal vector. In the middle panel, positive values $\left(-\mathrm{V}_{2}>0\right)$ indicate upward velocity. For the time period of this figure, positive $\mathrm{V}_{\mathrm{y}}$ is approximately in the poleward direction. The label DH denotes the interval of density hole whereas the label DP denotes the interval of density pile-up. 
density fluctuations around $2227 \mathrm{UT}$, the ion density decreased smoothly to $3 \times 10^{4} \mathrm{~cm}^{-3}$, which appeared to be the ambient density level in the nightside.

In Fig. 2 three dotted verical lines are drawn to separate the intervals of density hole (DH) and density pile-up (DP). We define density pile-up (DP) as a density structure with density enhancement and fluctuations. The annotations shown at the bottom of Fig. 2 indicate that the DH and DP structures were detected roughly in $1900-2100$ LT. The density hole was measured at geographic latitude $\left(-19.9^{\circ}\right.$ to $\left.-25.4^{\circ}\right)$ and geographic longitude $\left(311^{\circ}\right.$ to $\left.322^{\circ} \mathrm{E}\right)$. Adjacent to the $\mathrm{DH}$, the density pile-up was measured at geographic longitude $\left(322^{\circ}\right.$ to $\left.339^{\circ} \mathrm{E}\right)$. The region covered by the DH and DP structures is thus located inside the SAA general region, which spans approximately from $280^{\circ}$ to $360^{\circ} \mathrm{E}$ longitudes and between $10^{\circ}$ and $40^{\circ} \mathrm{S}$ latitudes (Langel 1987). In particular, we note that the $\mathrm{DH}$ left edge near $-20^{\circ}$ latitude and $311^{\circ} \mathrm{E}$ longitude is very close to our estimate of the SAA center at $630 \mathrm{~km}\left(-22^{\circ}\right.$ latitude and $307^{\circ} \mathrm{E}$ longitude) according to the IGRF magnetic field model for year 2000.

The two cross-track ion velocity components $\mathrm{V}_{\mathrm{y}}$ and $\mathrm{V}_{z}$ measured by the two ion drift meters are presented in the middle and bottom panels of Fig. 2 , respectively. In the spacecraft coordinate, a positive $\mathrm{V}_{2}$ approximately points toward the center of Earth, whereas the positive $\mathrm{V}_{\mathrm{y}}$ velocity is in the direction anti-parallel to the orbital plane normal vector. For the pass shown in Fig. 2 when ROCSAT-1 was at its maximum southern latitude, a positive $V_{y}$ velocity was approximately in the southward direction. Because the ROCSAT-1 orbit has an inclination angle of $35^{\circ}$, ion velocities both parallel and perpendicular to the magnetic field may contribute to the $\mathrm{V}_{y}$ and $\mathrm{V}_{z}$ measurements.

Figure 2 indicates that $\mathrm{V}_{\mathrm{z}}$ is small inside the density hole and upward inside the density pile-up (middle panel, Fig. 2). On this orbit upward velocity appeared to be appreciable in the $\mathrm{DP}$ region whereas upward velocity was negligible in the DH region. The bottom panel shows a large positive $\mathrm{V}_{\mathrm{y}}$ velocity inside both the $\mathrm{DH}$ and DP density structures, indicating large poleward drift velocities in these regions. Inside the DP region, the $V_{y}$ velocity component had large fluctuations similar to the density fluctuations detected by the ion trap sensor (top panel, Fig. 2).

Figure 3 summarizes the longitudinal density profile measured by ROCSAT-1 during the MADS event. A letter from $a$ to $g$ is assigned to each orbit. The ion density is plotted versus longitude from $280^{\circ} \mathrm{E}$ to $20^{\circ} \mathrm{E}$. According to Dst index (see Fig. 1), the magnetic storm main phase just started (2040 UT) during orbit $a$ and the measured ion density displayed a typical density variation with a small enhancement approximately at $330^{\circ} \mathrm{E}$ longitude as ROCSAT-1 moved from dayside to nightside (curve $a$, Fig. 3). However, on the next six orbits (orbit $b$ through orbit $g$ ), density holes and density pile-ups were observed. In Fig. 3 the interval of a density hole is marked by a dashed line, whereas the interval of a density pile-up is marked by a solid line. The ion density data described in Fig. 2 is presented in Fig. 3 as orbit $b$. For orbit $b$ ion density initially varied gradually from $10^{6} \mathrm{~cm}^{-3}$ to about $3 \times 10^{5} \mathrm{~cm}^{-3}$ as longitude varied from $280^{\circ}$ to about $311^{\circ} \mathrm{E}$. After $311^{\circ} \mathrm{E}$ longitude, ion density decreased rapidly by a factor of 9 to about $4 \times 10^{4} \mathrm{~cm}^{-3}$. A region of enhanced density with $\sim 10^{6} \mathrm{~cm}^{-3}$ immediately followed the density hole. The density hole on orbit $b$ had a longitudinal width of $11^{\circ}$, and the following density pile-up had an even larger longitudinal extent of $17^{\circ}$. Using a satellite speed of 7.5 $\mathrm{km} / \mathrm{s}$, we estimate that the density hole spanned a distance of about $1470 \mathrm{~km}$ along the satellite 


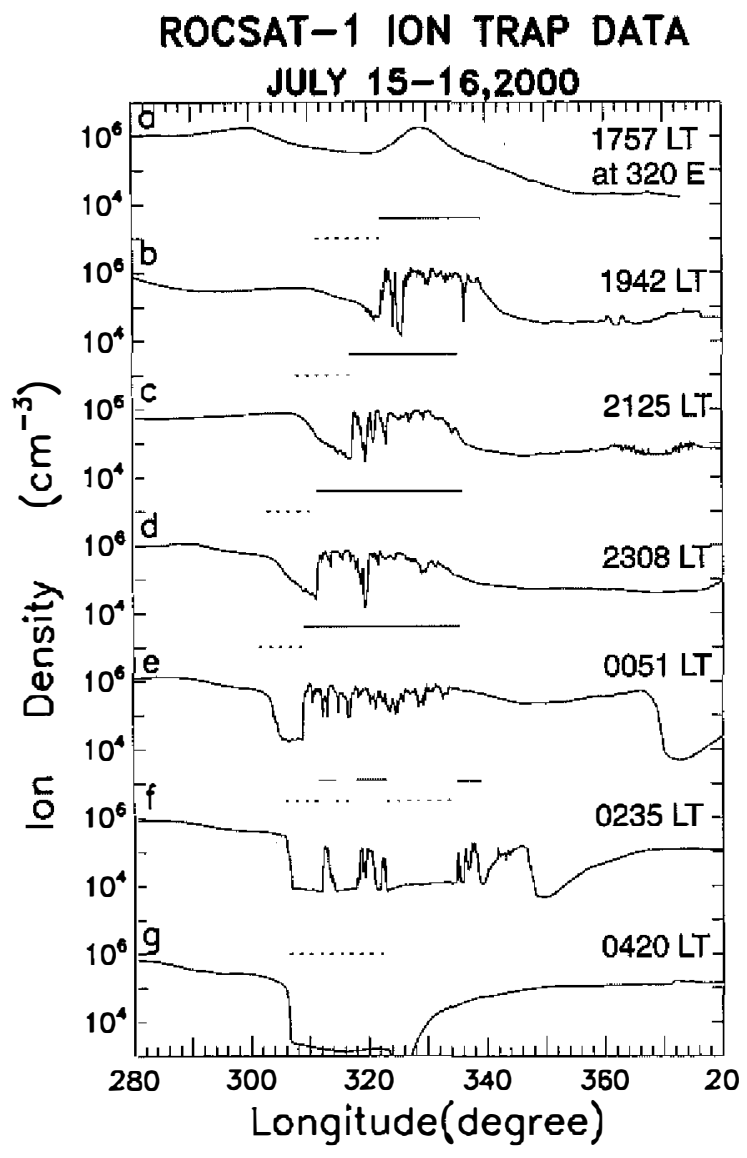

Fig. 3. Total ion density measured by the ROCSAT-1 ion trap sensor versus geographic longitude for seven successive orbits over the South Atlantic Anomaly on July 15-16, 2000. Each orbit is labeled with a letter from $a$ to $f$. The dashed line marks the density hole region, whereas the solid line marks the density pile-up region. The local time at $320^{\circ} \mathrm{E}$ longitude is shown on the right hand side for each orbit.

path. Similarly the spatial size of the density pile-up is estimated to be $2110 \mathrm{~km}$. The largescale density structures observed on orbits $c, d$ and $e$ are similar to those observed on orbit $b$.

On orbit $e$, a large smooth density depletion was measured at about $13^{\circ}$ East longitude when local time was near $0438 \mathrm{LT}$ (curve $e$ in Fig. 3). On the next orbit, a similar density depletion was again observed at about $350^{\circ}$ East longitude (curve $f$ ) and its local time was found to remain around $0444 \mathrm{LT}$. These pre-dawn density depletions, which might involve sunlit chemistry, have been studied by Su et al. (2000). They appear to be unrelated to the magnetic anomaly density structures reported here.

The ROCSAT- 1 observations during orbit $f$ are very interesting because several density holes and density pile-ups had been sequentially detected along the satellite path. Since the first two density pile-ups were sandwiched on both sides by density holes, they might be considered as a detached density pile-up. The first detached DP was detected for a period of 49 $\mathrm{s}$, while the second detached DP was observed for $95 \mathrm{~s}$. With only single satellite measurements, we could not distinguish whether they represent a spatial structure or a temporal variation.

To display the locations of density structures, we present ion density in color scale along the satellite track in Fig. 4, which takes about 8 hour ion trap data to construct the plot. Figure 4 shows that the deepest density hole was detected near the equator during the magnetic storm 


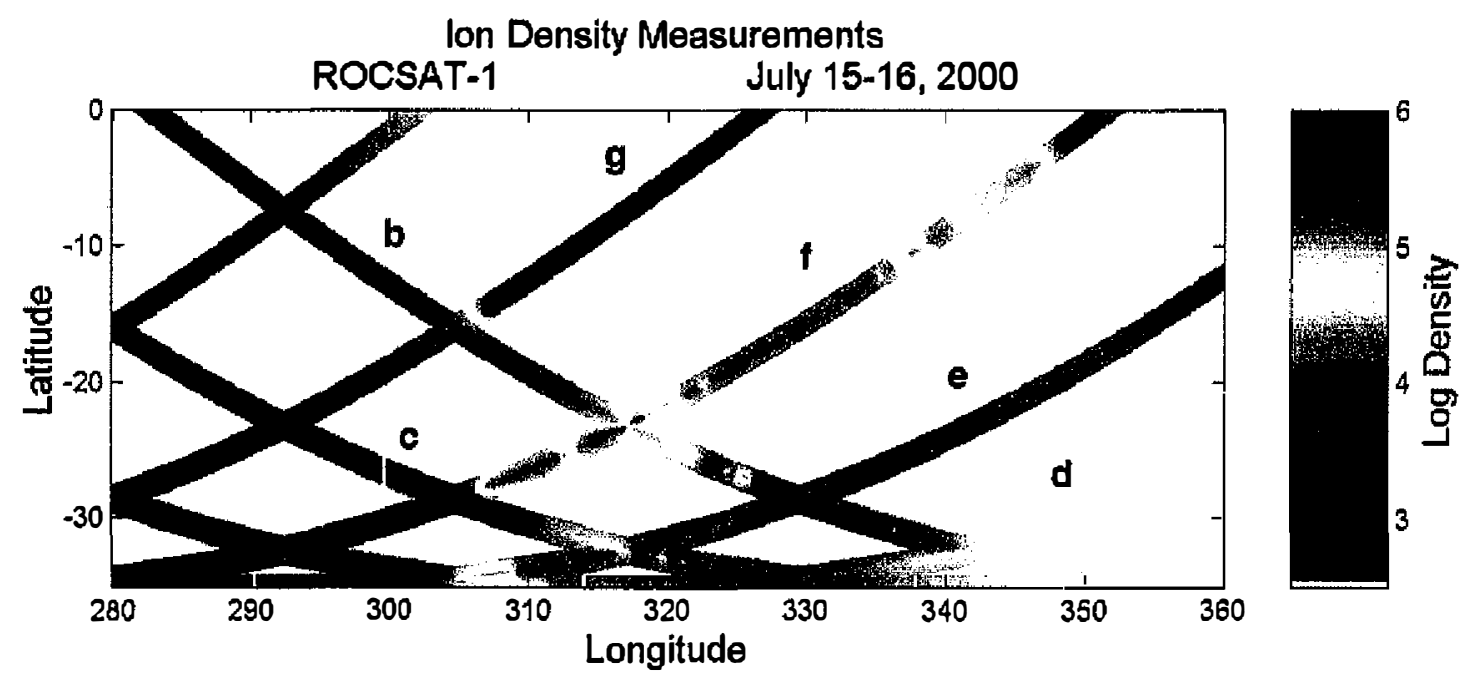

Fig. 4. Color plot of logarithmic density along the ROCSAT-1 satellite track. The figure is composed from ion density measurements during six orbits from orbit $b$ to orbit $g$.

recovery phase (orbit $g$ ). On this orbit, ROCSAT-1 crossing equatorward of the SAA observed a density hole without an adjacent density pile-up. Unlike the density holes observed at off-equator latitudes, ion drift velocity had been found to be very turbulent inside this density hole (orbit $g$ ), according to a separate study by Yeh et al. (2000). Their results also indicate that the plasma properties of the equatorial density depletion were different from those of low latitude density holes (e.g., on orbits $b, c$, and $\boldsymbol{d}^{\text {) }}$ and might contain heavy $\mathrm{NO}^{+}$ions.

In all passes density pile-ups were always detected after density holes( i.e., eastward of the density holes). Figure 3 shows that density pile-ups were superimposed by density irregularities, which were absent inside density holes. Examining ion drift meter data, we note that ion drift velocity also had large fluctuations inside density pile-ups (e.g., see Fig. 2).

We summarize the observations of magnetic anomaly density structures (MADS) in Table 1 , which lists for each orbit the start time of density hole, the mean Dst for the observation interval, the DH spatial size $\mathrm{L}_{\mathrm{DH}}$, the DP spatial size $\mathrm{L}_{\mathrm{DP}}$, and the density ratio $\mathrm{n}_{\mathrm{O}} / \mathrm{n}_{\mathrm{DH}}$. Here $\mathrm{n}_{\mathrm{O}}$ is the ambient density and $n_{D H}$ is the minimum density inside a density hole. Table 1 indicates that ion density in general dropped by a factor of 10 to 45 from the ambient to the bottom of density hole. Excluding the DH and DP adjacent to the detached density pile-ups (orbit $f$ ), we determine that density hole had a size varying from $800 \mathrm{~km}$ to $1800 \mathrm{~km}$ and density pile-up had a size varying from $2000 \mathrm{~km}$ to $3100 \mathrm{~km}$ along the satellite path. The spatial scales of density holes and density pile-ups are therefore much larger than the typical size of equatorial density bubbles. The MADS on orbits $b, c$, and $\boldsymbol{d}$ were detected when magnetic storm activity was most intense (Dst was between -250 and -300). ROCSAT-1 observed the remaining MADS (orbits $e, f$ and $g$ ) during the storm recovery phase (Dst $=-200$ ).

To discern the local time effects, we plot the magnetic latitude of density structures as a 
Table 1. ROCSAT-1 Observations of Magnetic Anomaly Density Structures.

\begin{tabular}{ccrrrr}
\hline Orbit & $\mathrm{UT}^{*}$ & $\mathrm{Dst}$ & $\mathrm{L}_{\mathrm{DH}}{ }^{+}$ & $\mathrm{L}_{\mathrm{DP}}{ }^{\$}$ & $\mathrm{~N}_{\mathcal{O}} / \mathrm{n}_{\mathrm{DH}}{ }^{\#}$ \\
$\mathrm{~b}$ & 2219 & -300 & 1470 & 2110 & 9 \\
$\mathrm{c}$ & 0002 & -280 & 1060 & 2010 & 20 \\
$\mathrm{~d}$ & 0144 & -270 & 910 & 2630 & 18 \\
$\mathrm{e}$ & 0327 & -250 & 820 & 3100 & 30 \\
$\mathrm{f}$ & 0511 & -200 & 720 & 370 & 45 \\
$\mathrm{f}$ & 0513 & -200 & 470 & 710 & 39 \\
$\mathrm{f}$ & 0516 & -200 & 1750 & 630 & 18 \\
$\mathrm{~g}$ & 0655 & -180 & 2640 & - & 35 \\
\hline
\end{tabular}

* UT is the start time of density hole.

$+L_{D H}$ is the spatial size of density hole.

$\$ L_{D P}$ is the spatial size of density pile-up.

$\# \mathrm{n}_{\mathrm{O}} / \mathrm{n}_{\mathrm{DH}}$ is the ratio of the ambient density $\mathrm{n}_{\bullet}$ over $\mathrm{n}_{\mathrm{DH}}$, the minimum density inside the density hole.

function of local time (Fig. 5). Note that ROCSAT-1 observed MADS in the nightside from 1900 to $0400 \mathrm{LT}$. The position of the SAA center at the beginning of density hole for each orbit is marked with + in Fig. 5. Using the IGRF magnetic field model, we determine the SAA center by locating the magnetic field minimum at the ROCSAT- 1 altitude of $630 \mathrm{~km}$ for year 2000. The SAA center is found to be located at $-12^{\circ}$ magnetic latitude and $18^{\circ} \mathrm{E}$ magnetic longitude. Figure 5 clearly indicates that the observed MADS co-rotated with the SAA. This figure also shows that the density structures observed in the pre-midnight sector were confined in longitude.

To further study the relationship of MADS and the SAA, we plot the satellite trajectory during the MADS event and three contours of constant magnetic field strength in the geomagnetic coordinate (Fig. 6). Density structures were detected inside the contour of magnetic field strength 0.195 guass, and the density holes were generally close to the SAA center. Note that the minimum magnetic field strength at the SAA center is about 0.18 guass. Based on this figure, we construct a schematic illustrating the morphology of density hole and density pileup (Fig. 7). The DH and DP regions are obtained by connecting the edges of sharp density gradient. Figure 7 illustrates that the anomalous density structures were confined within $30^{\circ}$ in longitude east of the SAA center.

\section{DISCUSSION}

The ROCSAT 1 satellite observed the unusual features of low latitude density structures when it happened to fly directly over the SAA region during the magnetic storm of July 15-16, 2000. Large scale density holes and density pile-ups detected by ROCSAT-1 were found to be confine near the SAA and co-rotate with the SAA. Due to its low inclination orbit the 


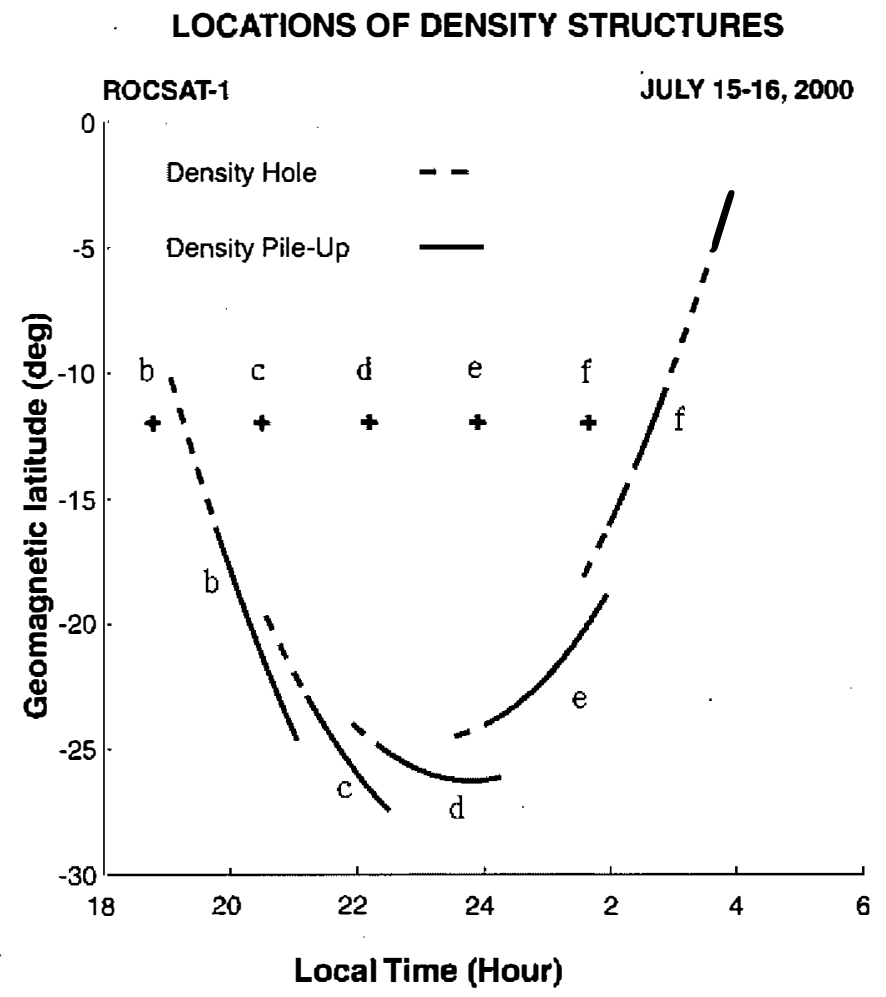

Fig. 5. Satellite path of magnetic anomaly density structures. The vertical axis is geomagnetic latitude and the abscissa is local time. The + symbol indicates the position of the SAA center at the beginning of the density hole observation on each orbit.

ROCSAT- 1 satellite was able to measure the longitudinal profile of the magnetic anomaly density structures that has not been obtained before.

Previously large scale ion depletions have been observed only by DMSP satellite during severe magnetic storms. (Greenspan et al. 1991; Foster and Rich 1998). The magnetic anomaly density holes observed by ROCSAT-1 at low latitudes are similar to the equatorial density depletions observed by DMSP F9 over South America during the great magnetic storm of 1989 (Greenspan et al. 1991). The equatorial density depletion event observed by DMSP had a density depletion extended over $4500 \mathrm{~km}$ along the DMSP path from a magnetic latitude of $9^{\circ}$ to a magnetic latitude of $-16^{\circ}$. We note that the plasma properties of density holes measured by ROCSAT- 1 were about the same as those of equatorial density depletions measured by DMSP, which measured a density dropout by a factor of 50 and an ion density about $10^{4} \mathrm{~cm}^{-3}$. At the southern edge of the equatorial density depletion, DMSP detected transient density increases, which appeared to be similar to density pile-up structures observed by ROCSAT-1 as well.

It is important to point out the significant differences between the ROCSAT-1 and DMSP observations. Inside the equatorial density depletion region DMSP detected large upward drifts, 
whereas ROCSAT-1 observed negligible upward velocity inside the density holes. The different feature of upward velocity might be attributed to the fact that ROCSAT- 1 detected density depletions close to the SAA and DMSP detected them closer to the equator. Inside the density holes ROCSAT-1 measured large poleward drift, which could not be measured by DMSP because its ion drift meter instrument could not measure ion drift velocity in the meridian direction. In addition Greenspan et al. (1991) reported that DMSP observed equatorial density depletions as far west as $-8^{\circ}$ magnetic longitude, much further away from the SAA than the MADS event studied here. ROCSAT- 1 measured density holes mainly between $10^{\circ}$ and $45^{\circ}$ magnetic longitude, corresponding to between $305^{\circ}$ and $340^{\circ}$ geographic longitude. It seems that ROCSAT-1 detected density holes near the SAA central region whereas DMSP observed density depletion in the west outskirt of the SAA. The significance of the longitudinal difference will be further studied.

Greenspan et al. (1991) attributed the drastic equatorial ion density depletion to a strong

LOCATIONS OF MADS

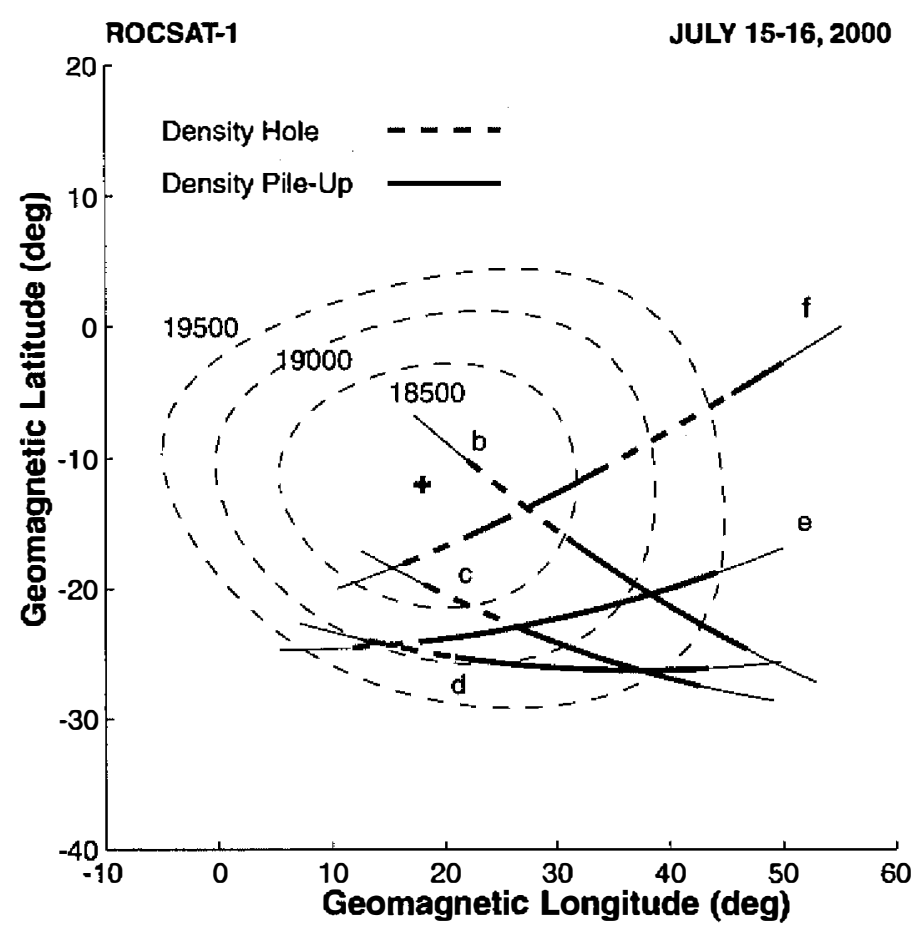

Fig. 6. Locations of magnetic anomaly density structures (MADS) in the coordinate of magnetic latitude and magnetic longitude. Three contours of magnetic field strength from 18500 to $19500 \mathrm{nT}$ around the SAA center are plotted. The SAA center is marked by + . The solid line segment indicates the interval when DP was detected, whereas the dashed line shows the interval of detecting DH. The thin line represents the satellite trajectory outside MADS. 
SCHEMATICS OF MADS

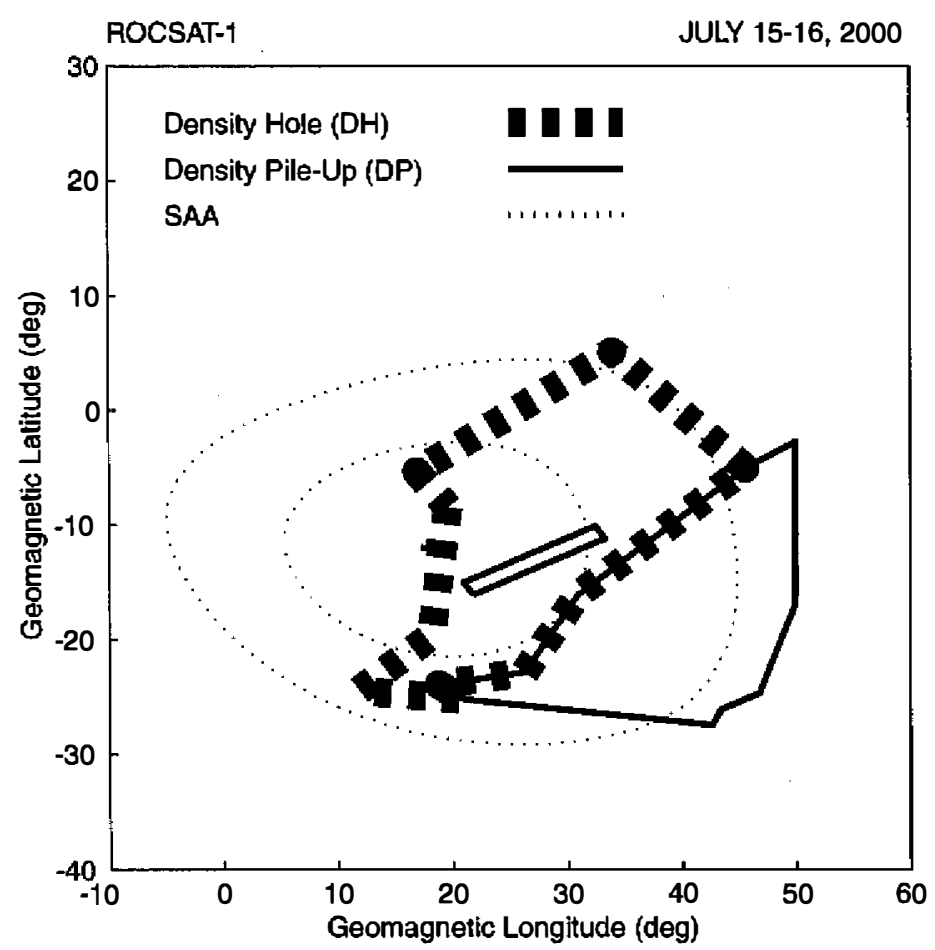

Fig. 7. Schematic illustrating the relative position of density hole and density pile-up. The small isolated square represents a detached density pile-up.

enhancement of the equatorial ion fountain by an eastward electric field in the dusk sector. They suggested that density holes are probably produced as eastward electric fields at low latitudes are enhanced during the storm and cause the F2 peak layer to rise. Normally the F region peak lies below the satellite and the density decreases sharply toward lower altitudes below the F region peak. As the F2 peak moves upward, a satellite would generally detect a density increase. Greenspan et al. (1991) argued that the enhanced eastward electric fields under severe magnetic storm conditions might be sufficiently strong to lift upward the entire $F$ region peak above the satellite altitude. In this case a low altitude orbiting satellite would detect ion density depletion. However the ion velocity measurements of ROCSAT-1 indicate that no appreciable ion upward motion was detected inside the density holes (see Fig. 2). The ROCSAT-1 measurements thus imply that the F2 peak layer did not rise to the satellite altitude of $630 \mathrm{~km}$ at the magnetic latitudes of density holes (between $-10^{\circ}$ and $-24^{\circ}$ ). Nevertheless we could not rule out the possibility that the F2 peak layer near the equator drifted to higher altitudes above the satellite.

On the other hand the ion upward drift observed in the density pile-up region should raise the F2 peak layer to higher altitudes. Since density pile-ups had a high density level and an ion upward drift velocity, it is likely that the F2 peak layer was raised above the satellite altitude 
$(630 \mathrm{~km})$ inside the density pile-up region. Furthermore, the presence of large density and velocity fluctuations inside density pile-ups probably implies that ROCSAT-1was traversing the bottom side of the F2 peak layer, because the bottom side of the F2 peak layer is generally very unstable to upward drifts (Kelley 1989).

Since no appreciable ion upward motion was detected inside the density holes, the F2 peak layer should not rise to higher altitudes inside the density hole region. Therefore the main difference between a density hole and a density pile-up is in the vertical height of the F2 peak layer, which is interpreted to be higher in the density pile-up region than in the density hole region. The strong ion poleward motion inside the density hole probably had depleted ions above the topside ionosphere from the poleward side of density hole. Inside the density pileup, however, the ion poleward velocity was reduced considerably and at the same time the upward motion became prevalent (see Fig. 2), causing the high density F2 peak layer to rise to higher altitudes. As a result, a much higher density would be detected by ROCSAT-1 in the density pile-up region.

The observed sequence of density holes suggests that the density hole might have first occurred poleward of the SAA and then expanded equatorward and eastward. For example, the first density hole was observed at $-25^{\circ}$ geographic latitude and no density depletion had been detected at latitudes between $0^{\circ}$ and $-25^{\circ}$ on orbit $b$ (see Figs. 2 and 4). Later on orbit $g$ a large density hole was detected at latitude between $0^{\circ}$ and $-15^{\circ}$ with comparable longitudes (Fig. 4). Furthermore the density holes detected on orbit $f$ extended considerably eastward of the density holes detected on earlier orbits (Figs. 4 and 6).

On the initial orbits (orbits $b, c$, and $d$ ) the density holes were found to be V-shaped with a gradual gradient on the left hand side and a sharp boundary on the right hand side. The density profile became $\mathrm{U}$-shaped on the following orbits. Thus the density hole was initially narrow and then became wider on subsequent orbits, suggesting that the ion depletion expanded with time. The density level inside the density hole also decreased with time. For example, the density level inside the density hole varied from about $4 \times 10^{4} \mathrm{~cm}^{-3}$ on orbit $b$ to $10^{3} \mathrm{~cm}^{-3}$ on orbit $g$. Thus the density holes observed during the storm recovery phase appeared to be deeper than the earlier density holes.

The poleward and upward ion motions inside the observed MADS imply the presence of eastward electric fields at low latitudes. During magnetic storm disturbances, magnetospheric electric fields can penetrate to low latitudes due to either ionospheric disturbance dynamo or prompt response to sudden changes in the polar cap potential, according to the empirical models of storm time equatorial zonal electric fields (Spiro et al. 1988; Fejer and Scherliess 1997; Scherliess and Fejer 1997; Blanc and Richmond 1980; Richmond 1995). In the first mechanism the short-term ionospheric disturbance dynamo action due to an enhanced energy input into the high-latitude ionosphere drives electric fields at low latitudes with a time delay of about 1-12 hours (Scherliess and Fejer 1977). In this empirical ionospheric disturbance dynamo model, the zonal electric field is eastward at night with largest amplitudes near sunrise without regard to the storm phase. In the second mechanism, the prompt peneration electric fields associated with an increase in the polar cap potential are westward in the nightside and have a decay time constant of about 1 hour (Fejer and Scherliess 1997). However, during the storm recovery phase, the prompt penetration of magnetospheric electric fields produces east- 
ward electric fields because of decreases in the polar cap potential. Since the ion motion associated with magnetic anomaly density structures was upward, the zonal electric fields causing the observed MADS should be eastward. Because the MADS on the first three orbits occurred during the storm main phase, the eastward zonal electric field producing these MADS could only be caused by the short-term ionospheric disturbance dynamo. However, on the last three orbits during the storm recovery phase, the eastward zonal electric fields responsible for the production of MADS might be attributed to both the prompt penetrating magnetospheric electric fields and the zonal electric fields produced by the ionospheric disturbance dynamo.

In this paper we provide clear evidence that MADS co-rotated with the SAA. Because of the widening of the electron loss cone, eastward drifting electrons are expected to be depopulated below $300 \mathrm{~km}$ and $\mathrm{L}=1.3$ as energetic electrons pass through the SAA region of weakened magnetic field strength (Roederer et al. 1967). Several satellites have observed energetic electron precipitation fluxes in the SAA region (Vampola and Gorney 1983; West and Buck 1976). During a severe magnetic storm, intense energetic electron precipitation could occur over the SAA, producing X-ray ionization and conductivity enhancement in the nighttime E region (Paulikas 1975; Jayanthi et al. 1997). During the great storm of March 1989, near-equatorial ground-based ionosondes in Brazil recorded $E$ layer enhancement due to particle precipitation near the magnetic anomaly (Batista et al. 1991). Greenspan et al. (1991) described a likely scenario in which conductivity gradients at the edge of the SAA would cause the development of eastward polarization electric fields. Following this scenario, we suggest that energetic electron precipitation might have occurred over the region of magnetic anomaly density structures, producing ionospheric conductivity gradients and eastward polarization electric fields in a localized area. The localized polarization electric fields are superimposed on the storm time zonal electric fields that have already penetrated to low latitudes in the nighttime sector. Therefore the localized polarization electric fields could be the third source of eastward electric fields causing MADS in addition to the zonal electric fields due to the prompt penetration of magnetospheric electric fields and the ionospheric disturbance dynamo.

During geomagnetic disturbances, the electric fields and particle populations characterizing the auroral region expand equatorward, and their effects appear at previously sub-auroral latitudes (Spiro et al. 1978; Yeh et al. 1991; Foster et al. 1998; Burke et al. 2000). It is possible that density depletion observed by ROCSAT-1 near the SAA latitudes might be due to the low latitude penetration of mid-latitude density trough, which corresponds to the outer boundary of the plasmasphere at the topside ionosphere. The mid-latitude density trough, which maps to the plasmapause in the magnetosphere, is associated with the boundary between the co-rotating inner magnetosphere and strong convection electric fields driving the ionospheric circulation at auroral latitudes. Under very intense geomagnetic activities as in the case of the July 2000 storm, the penetration of magnetospheric electric fields may have carried the mid-latitude trough equatorward to the SAA latitude $(L=1.3)$. Foster and Rich (1998) have previously reported such low latitude observations of mid-latitude density trough near the SAA region.

Yeh et al. (2000) used the ROCSAT-1 IPEI data to study prominent depletion structures at magnetic latitudes $14^{\circ}-20^{\circ} \mathrm{S}$ in the pre-dawn hours when the storm was in its early recovery 
phase. These prominent density depletion structures were characterized by large-amplitude density decrease $\left(\mathrm{N} / \mathrm{N}_{\mathrm{o}}<1 \%\right)$ with rapid horizontal ion drifts. These density depletions were observed at the same magnetic latitudes as the magnetic anomaly density holes reported here. However, these density depletion structures had large amplitude irregularities, whereas magnetic anomaly density holes lacked of irregularities. Nevertheless both phenomena might be related to the effects of enhanced eastward electric fields due to the storm-time disturbances.

The ROCSAT- 1 observations during orbit $f$ are very interesting because two detached density pile-ups were detected (see Fig. 3). A single satellite density profile is usually insufficient for determining whether the detached density pile-up density profile represents an isolated spatial structure like density islands or evidence of temporal variations due to a boundary oscillation of a density pile-up. Our preliminary study of ion drift patterns nevertheless gives a hint that they are probably caused by the horizontal motion of a density pile-up.

In summary we present direct observation evidence relating the occurrence of ionospheric density structures with the SAA during a very intense magnetic storm. It is generally believed that energetic particle precipitation occurring in the region of density holes and density pileups might be able to increase ionospheric conductivity and conductivity gradient across the boundary, and produce eastward polarization electric fields localized in the precipitation region. We suggest that the combination of localized polarization electric fields and magnetospheric storm time zonal electric fields associated with the penetration of magnetospheric electric fields in the nightside might produce the complicated features of magnetic anomaly density structures at the topside ionosphere near the SAA. The ROCSAT- 1 observations imply that such drastic space weather effects might be initiated poleward of the SAA and then expanded equatorward with time. The role of the SAA in affecting the ionospheric disturbance dynamo and the penetration of magnetospheric electric fields needs to be further investigated to better understand magnetic anomaly density structures.

Acknowledgments The author CSL would like to thank the hospitality of Institute of Space Science, National Central University where he conducted the present study. We thank R. A. Heelis and L. C. Lee for their useful comments. We also thank J. M. Wu, S. C. Yang and P. F. Shieh for their assistance in data processing. The work has been supported by National Space Program Office Operation Contract NSC89-NSPO(A)-PDD-008-STP01 and National Science Council Research Project NSC-89-2111-M-008-053 of Taiwan.

\section{REFERENCES}

Batista, I. S., E. R. de Paula, M. A. Abdu, and N. B. Trivedi, 1991: Ionospheric effects of the March 13, 1989, magnetic storm at low and equatorial latitudes. J. Geophys. Res., 96, 13,943-13,951.

Blanc, M., and A. D. Richmond, 1980: The ionospheric disturbance dynamo. J. Geophys. Res., 85, 1,669-1,686.

Burke, W. J., A. G. Rubin, N. C. Maynard, L. C. Gentile, P. J. Sultan, F. J. Rich, O. de La Beaujardiere, C. Y. Huang, and G. R. Wilson, 2000: Ionospheric disturbance observed by DMSP at middle to low latitudes during the magnetic storm of June 4-6, 1991. J. 
Geophys. Res., 105, 18,391-18,405.

Chang, Y.-S., W.-I. Chiang, S.-J. Ying, B. J. Holt, C. R. Lippincott, and K.-C. Hsieh, 1999: System Architecture of the IPEI payload on ROCSAT-1. TAO supplementary issue, 718.

Fejer, B. G., and L. Scherliess, 1995: Time dependent response of equatorial ionospheric electric fields to magnetospheric disturbances. Geophys. Res. Lett., 22, 851-854.

Fejer, B. G., and L. Scherliess, 1997: Empirical models of storm time equatorial zonal electric fields. J. Geophys. Res., 102, 24,047-24,056.

Foster, J. C., S. Cummer, and U. S. Inan, 1998: Midlatitude particle and electric field effects at the onset of the November 1993 geomagnetic storm. J. Geophys. Res., 103, 26,359$26,366$.

Foster, J. C., and F. J. Rich, 1998: Prompt midlatitude electric field effects during severe geomagnetic storms. J. Geophys. Res., 103, 26,367-26,372.

Greenspan, M. E., C. E. Rasmussen, W. J. Burke, and M. A. Abdu, 1991: Equatorial density depletions observed at $840 \mathrm{~km}$ during the great magnetic storm of March 1989. J. Geophys. Res., 96, 13,931-13,942.

Jayanthi, U. B., M. G. Pereira, I. M. Martin, Y. Stozkov, F. D'Amico, and T. Villela, 1997: Electron precipitation associated with geomagnetic activity: Balloon observation of $\mathrm{X}$ ray flux in South Atlantic Anomaly. J. Geophys. Res., 102, 24,069-24,077.

Kelley, M. C., 1989: The Earth's Ionosphere, Academic Press.

Langel, R. A., 1987: The Main Field, in Geomagnetism. In: J. A. Jacobs (Ed.), Vol. 1, 249492, Academic Press.

Luhmann, J. G., and A. L. Vampola, 1977: Effects of localized sources on quiet time plasmasphere electron precipitation. J. Geophys. Res., 82, 2,671-2,675.

Paulikas, G. A., 1975: Precipitation of particles at low and middle latitudes. Rev. Geophys., 13, 709-734.

Richmond, A. D., 1995: Ionospheric electrodynamics, in Handbook of Atmospheric Electrodynamics. In: H. Volland (Ed.) Vol. 2, , Pp. 249-290, CRC Press, Boca Raton, Fla..

Roederer, J. G., J. A. Welch, and J. V. Herod, 1967: Longitude dependence of geomagnetically trapped electrons. J. Geophys. Res., 72, 4,431-4,447.

Scherliess, L., and B. G. Fejer, 1997: Storm time dependence of equatorial disturbance dynamo zonal electric fields. J. Geophys. Res., 102, 24,037-24,046.

Spiro, R. W., R. A. Heelis, and W. B. Hanson, 1978: Ion convection and the formation of the mid-latitude F region ionization trough. J. Geophys. Res., 83, 4,255-4,264.

Spiro, R. W., R. A. Wolf, and B. G. Fejer, 1988: Penetration of high-latitude electric field effects to low latitudes during SUNDIAL 1984. Ann. Geophys., 6, 39-50.

Su, S.-Y., H. C. Yeh, R.A. Heelis, J. M. Wu, S. C. Yang, and Y. Wu, 2000: Motions of large density structures in the nighttime topside ionosphere during the July 15-16, 2000 magnetic storm. EOS, F950.

Vampola, A. L., and D. J. Gomey, 1983: Electron energy deposition in the middle atmosphere. J. Geophys. Res., 88, 6,267-6,274. 
West, H. I., and R. M. Buck, 1976: Energetic electrons in the inner belt in 1968. Planet. Space Sci., 24, 643-655.

Yeh, H.-C., J. C. Foster, F. J. Rich, and W. Swider, 1991: Storm time electric field penetration observed at mid-latitude. J. Geophys. Res., 96, 5,707-5,721.

Yeh, H. C.; S. Y. Su, Y. C. Yeh, J. M. Wu, R. A. Heelis, and B. J. Holt, 1999: Scientific mission of the IPEI payload on board ROCSAT-1. TAO supplementary issue, 19-42.

Yeh, H. C., S.-Y. Su, R. A. Heelis, and W. R. Coley, 2000: Molecular ions observed by ROCSAT-1 at 600-km astitude in low-latitude ionosphere. EOS, F946.

Yeh, H. C., S. Y. Su, and R. A. Heelis, 2001: Storm time plasma irregularities in the pre-dawn hours observed by the low-latitude ROCSAT-1 satellite at $600 \mathrm{~km}$ altitude. Geophys. Res. Lett., 28, 685-688. 